Axel W. Bauer: Zur Einführung der naturwissenschaftlichen Methode in die Medizin. In: Modelle der Pathologischen Physiologie. Hrsg. von Wilhelm Doerr und Heinrich Schipperges. Heidelberger Akad. der Wissenschaften. Berlin (u.a.) 1987. S. 41-53.

\title{
1.2 Zur Einführung der naturwissenschaftlichen Methode in die Medizin
}

Axel Bauer

\section{Einführung}

Mitten im Biedermeier, jener im Bereich der Heilkunde von den Epigonen der Naturphilosophie und den Vertretern der Naturhistorischen Schule geprägten Epoche, beginnt in Deutschland eine neue Phase der Medizin, welche wir heute als die „naturwissenschaftliche“ charakterisieren. Das Vorfeld zu dieser Entwicklung finden wir bereits am Ende der zwanziger Jahre des 19. Jahrhunderts angelegt, wenn auch zunächst mehr in programmatischen Reden als im praktischen Handeln der Wissenschaftler.

Als Forum für grundsätzliche Beiträge zur Wissenschaftstheorie eignen sich besonders die alljährlichen Versammlungen Deutscher Naturforscher und Ärzte, deren Intentionen sehr bald über den in den Statuten von 1822 festgelegten Hauptzweck (,den Naturforschern und Aerzten Deutschlands Gelegenheit zu verschaffen, sich persönlich kennen zu lernen") hinausreichen. Der Erste Geschäftsführer der 8. Versammlung, die 1829 in Heidelberg tagt, der Anatom und Physiologe Friedrich TIEDEMANN (1781-1861), formuliert die Aufgabe der Gesellschaft sehr präzise in seiner Eröffnungsrede:

„Was uns zusammen führte, ist allein das Suchen nach Wahrheit und der Trieb, unsere Kenntnisse zu erweitern, durch Mittheilung von Beobachtungen und Erfahrungen aus dem unermeßlichen Gebiete der Natur, durch Austausch von Ideen, und durch Erweckung und Anregung klarerer und tieferer Einsichten. Dadurch ..., daß gleich gesinnte ... Männer sich die Ergebnisse ihrer Forschungen mittheilen, werden neu errungene Thatsachen aufs schnellste in Umlauf gesetzt, ... Meinungen und Theorieen ... werden erwogen und geprüft, Zweifel und Einwürfe ... erhoben und beseitigt. Und so gelingt es, Blicke in die Geheimnisse der Natur und des Weltgebäudes zu thun"1.

Wahrheitssuche, Erkenntnistrieb, Beobachtung und Erfahrung, Ideenaustausch, Prüfung von Theorien, Einblick in die Geheimnisse der Natur - damit sind Schlüsselbegriffe für Motive, Methoden und Ziele der „Naturforscher“ genannt, gleichsam eine verbindende „Ideologie“ der ansonsten recht locker organisierten „Scientific Community“, die sich seit 1822 stets im September an wechselnden Orten Deutschlands versammelt. Getragen wird diese geistige Bewegung von einem schwungvollen Optimismus, einem ganz auf die Zukunft hin orientierten Selbstbewußtsein, das schon die Gegenwart weit über das Niveau der Vergangenheit zu erheben scheint. Wie sehr übertrifft doch unser Zeitalter hinsichtlich der physikalischen Kenntnisse jenes der Griechen und Römer, ruft TiEdEMANN aus. Die Methode der wissenschaftlichen Naturforschung sei den 
Alten kaum bekannt gewesen, nicht zuletzt wegen des Mangels an geeigneten Instrumenten wie Waagen, Wärmemessern, Barometern, Hygrometern, Mikroskopen oder Ferngläsern. Deshalb hätten sich die Vorfahren mit Spekulation und Metaphysik behelfen müssen, wobei aber nur Spitzfindigkeiten über die Entstehung der Welt und ihr Wesen das Ergebnis waren².

Die von TIEDEMANN genannten Instrumente zeigen den Weg, auf dem die „Hirngespinste“ allmählich durch „subjective Erkenntnis aus objectiver Anschauung" ersetzt werden sollen: quantifizierende Messung und optische Vergrößerung der physikalischen, chemischen oder biologischen Strukturen. Erst mit diesen Mitteln gelingt es, die Natur „durch Beobachtungen zu belauschen“, ja mehr noch, sie „durch Versuche zu befragen “3. TIEDEMANN bezeichnet die Gebiete, auf denen die Naturwissenschaft bereits zu hoffnungsvollen Ergebnissen gelangt ist: Astronomie, Chemie, Mineralogie, Geographie, Zoologie und Botanik, Vergleichende Anatomie, Physiologie und Naturgeschichte. Insbesondere die Ärzte seien mit ihrem praxisorientierten Verstand als Förderer des nüchternen Denkens und Gegner der Spekulation hervorgetreten. Der Redner sieht die Medizin mitten auf dem Weg zu einer wissenschaftlichen Disziplin, und dies um so mehr, je größer ihre Distanz zur Philosophie werde und je enger sie ihr Verhältnis zu den physikalischen Wissenschaften gestalte. Gleichzeitig leisteten die Naturwissenschaften einen unschätzbaren Beitrag zum Ausbau der bürgerlichen Gesellschaft und der allgemeinen Kultur, indem sie dazu aufforderten, "nach den Gesetzen der Vernunft und Sittlichkeit zu handeln"4.

Auf den Tag genau 60 Jahre später, am 18. September 1889, wird wiederum in Heidelberg eine Naturforscherversammlung eröffnet, nunmehr schon die zweiundsechzigste! Diesmal spricht der 1. Geschäftsführer GEORG HERMANN Quincke (1834-1924), Professor für Physik an der Ruperto-Carola, und er beginnt seine Rede mit einem Rückblick auf jene Tagung von 1829. Wievieles hat sich doch seither geändert! „Damals wurden die Theilnehmer der Versammlung von der Post befördert. Heute reisen wir auf der Eisenbahn mit mehr als vierfacher Geschwindigkeit"s. Während seinerzeit die Naturforscher nur über 6 Fachabteilungen verfïgt hätten, tage man heute in 32 Sektionen und mit vierfacher Teilnehmerzahl.

Doch nicht nur die äußeren Umstände haben sich verändert; weit dramatischer erscheint QUINCKE der Wandel in den Wissenschaften selbst. Voller Stolz konstatiert der Physiker: „Ueberall versuchte man in Deutschland die Methoden der Physik auf verwandte Gebiete zu übertragen, medicinische Fragen mit Hilfe der Naturwissenschaften zu lösen, aus dem Befunde der Leichen über den Verlauf der Krankheit und die Zweckmässigkeit der angewandten Heilmittel zu entscheiden und so in gemeinsamer Arbeit die beiden grossen Gebiete der Wissenschaft zu fördern, welche in unseren Versammlungen seit ihrem Bestehen in so glücklicher Weise vereinigt sind ... Man kann wohl sagen: zu dieser Zeit der ersten Heidelberger Naturforscher-Versammlung begann eine neue Epoche der Naturwissenschaft und Medicin in Deutschland"6. Soweit QuinCKE im Jahre 1889.

Tatsächlich trennt die beiden Heidelberger Naturforscherversammlungen mehr als nur jene quantitative Beschleunigung des Reisetempos von der Postkutsche zur Eisenbahn; besonders in der Medizin des 19. Jahrhunderts wird ein 
qualitativer Sprung deutlich, der von wissenschaftshistorischer Seite gelegentlich als Paradigmawechsel im Sinne KunNs, besser vielleicht als Konzeptwandel im Sinne Karl Eduard Rothschuns bezeichnet worden ist. Wie auch immer man diesen Prozeß benennen mag, stets spielt dabei die Einführung der "naturwissenschaftlichen Methode" in die Heilkunde eine wesentliche Rolle, das, was Quincke mit der „Übertragung der Methoden der Physik auf verwandte Gebiete" umschrieb. Ich möchte im folgenden versuchen, diese "naturwissenschaftliche Methode" und ihre Bedeutung für die Medizin des 19. Jahrhunderts darzustellen, nicht zuletzt aber auch ihre Grenzen, die gegen Ende des von mir betrachteten Zeitraums zum ersten Male deutlich werden.

\section{Von der Naturphilosophie zur Empirie}

Zur Zeit der Heidelberger Naturforscherversammlung von 1829 wird die Medizin in Deutschland noch weitgehend von den Epigonen der Naturphilosophie sowie von den Vertretern der sogenannten Naturhistorischen Schule beherrscht, als deren Begründer der Würzburger Kliniker JohanN Lukas ScHÖNLEiN (1793-1864) zu nennen ist. Beide Richtungen entwickelten eine Arbeitsweise, die auf den Prinzipien der Naturphilosophie Schellings basierte. FrIEDRICH WiLHelm SCHELLing (1775-1854) hat vor allem durch einige frühe Werke auf seine ärztlichen Zeitgenossen anregend gewirkt, und zwar durch die „Ideen zu einer Philosophie der Natur“ (1797), seinen „Ersten Entwurf eines Systems der Naturphilosophie“ (1799) sowie durch eine „Vorläufige Bezeichnung des Standpunktes der Medizin nach Grundsätzen der Naturphilosophie“ (1806). Geleitet von der Überzeugung der Einheit und Vernünftigkeit des Alls, entwarf SCHELLING ein System der Identität von Natur und Geist, von Realem und Idealem auf allen Ebenen und in allen Bereichen des Makrokosmos und des Mikrokosmos. Es gelte, die Natur aus dem Geist zu konstruieren, so sagte er 1797. Die Natur solle der sichtbare Geist, der Geist die unsichtbare Natur sein. Letztes Ziel des Naturphilosophen bleibe, die Idee der Natur zu erreichen, welche sich dem Geist als eine Stufung wachsender Vielfalt, als eine Steigerung offenbare. Natur ist dabei Fassade, ihr Inneres sind die Ideen, die sich in den Naturerscheinungen manifestieren.

Die einheitliche Abkunft und die Idealverwandtschaft aller Phänomene bedingen es, daß sich auf allen Stufen der Natur ein und dieselben Ideen, Grundprinzipien und Gesetze wiederfinden, nur dem Grad der Vollkommenheit nach unterschieden. Es muß daher das Streben der Naturforschung sein, auf allen Stufen der sich entwickelnden Natur diese gleichen Gesetzmäßigkeiten immer wieder aufzufinden. Den Weg dazu sieht SCHELLiNg im Aufspüren von Ähnlichkeiten und Verwandtschaften in den Eigenschaften und Kräften oder im Verhalten durch die Ermittlung von Analogien. Das Gemeinsame und die "wahre Idee" der Dinge zu begreifen, ist eine Sache des Sehers, nicht des Empirikers! „Einzelne waren und werden seyn, die der Wissenschaft nicht bedürfen, in denen die Natur sieht, und die selber in ihrem Sehen Natur geworden sind. Diese sind die waren Seher, die ächten Empiriker, zu denen die jetzt also sich nennenden sich verhalten, wie zu gottgesandten Propheten politische Kannengießer 
sich verhalten. Was durch einen solchen Seher verrichtet wird in Arzneikunst oder irgend einem andern Werk, das ist Wunder, denn es wird ohne Vermittlung erkannt und gethan" (Aphorismen ... 1805).

In der naturphilosophischen Methodologie spielen also Intuition und Analogiedenken eine führende Rolle; sie werden ergänzt durch die Prinzipien der Polarität und der Abstufung aller Lebenserscheinungen, an deren Spitze der Mensch als Zentralorganismus steht. Insbesondere der Analogieschluß entwikkelt sich unter Schellings Adepten zum Königsweg der philosophischen Naturkonstruktion; er rechtfertigt sich durch die Grundthese, daß der Organismus nichts anderes sei als die große Welt im kleinen (d.h. die Makrokosmos-Mikrokosmos-Analogie). Das Auffinden einer Analogie zwischen der Gliederung der Natur in die vegetative, die animalische und die sensitive Sphäre einerseits und dem Verhalten bestimmter Krankheiten andererseits genügt beispielsweise dem Arzt Dietrich Georg Kieser (1779-1862), um 1819 in seinem „System der Medizin" ein nosologisches Tableau zu postulieren, welches eine Einteilung nach Krankheiten der vegetativen, der animalischen und der sensitiven Organe vornimmt. Das aus allgemeinen Prinzipien, deren Gültigkeit axiomatisch vorausgesetzt wird, mittels der naturphilosophischen Methode Deduzierte bedarf weder eines experimentellen Beweises, - denn es genügt der Nachweis seiner Systemkonformität, etwa im Sinne einer offensichtlichen Analogie -, noch zumindest der empirischen Kontrolle am Krankenbett; nicht zuletzt ihre Praxisferne brachte die naturphilosophisch inspirierten Konzepte der Medizin denn auch schon bald in Verruf.

An dieser Situation konnte auch die vor allem in den zwanziger und dreißiger Jahren prosperierende, eher klinisch-empirisch orientierte Naturhistorische Schule wenig ändern, die sich um JOHANN LUKAS SCHÖNLEIN gruppierte. Immerhin lassen sich hier gewisse Modifikationen zur naturphilosophischen Heilkunde erkennen: Die streng empirisch arbeitende Schule ScHöNLEINS verzichtete auf eine a priori akzeptierte allgemeine Krankheitslehre. Durch genaue Beobachtung einzelner Krankheitsfälle wollte man stattdessen zur Konstruktion abstrakter Krankheitsbilder, zur Bildung von Krankheitsarten und -familien, endlich zu einem „natürlichen System“ der Krankheiten fortschreiten.

Da die Naturhistoriker um die Abstraktheit ihrer Krankheitseinheiten wußten, entwickelten sie Regeln für ihre Verfahrensweise, die sie nun wiederum den Vorstellungen der naturphilosophischen Medizin entnahmen. Die beiden wichtigsten Anleihen waren hierbei die Vorstellung, daß Krankheiten Prozesse seien, sowie die Überzeugung von der Örtlichkeit aller pathologischen Phänomene. Beobachtung und Empirie gewinnen mit der Naturhistorischen Schule zwar eine wachsende Bedeutung, doch kann sie ihren spekulativen Hintergrund in Gestalt naturphilosophischer Prämissen nicht verleugnen. Immerhin kehrt Schönlein das methodische Vorgehen der deduzierenden Naturphilosophen um, indem er induktiv vom Speziellen zum Allgemeinen fortschreitet. Der entscheidende Schritt zur experimentellen, quantifizierenden Überprüfung eines Sachverhalts fehlt allerdings noch gänzlich, die von großer Skepsis gegenüber mit Monopolanspruch auftretenden Theorien erfüllten Mediziner des Biedermeier schrecken vor abstrahierenden Überlegungen zurück. Zu deutlich hat man die Auswüchse der spekulativen romantischen Naturforschung noch vor Augen, deren "Ver- 
suche“, „Ahnungen", „Fragmente“ oder „Systeme“ zwar genialisch, aber ohne praktischen Nutzen waren. Die Kliniker der dreißiger Jahre beharren stattdessen auf dem Vordergründigen, Deskriptiven, der erfahrungsgemäßen Korrelation der Erscheinungen und Umstände. Man sucht nach empirischen Gesetzen, die möglichst numerisch gesichert sein sollen. KARL EDUARD ROTHSCHUH nannte das Zeitalter „vorexperimentell“ und „geradezu erfahrungssüchtig“7.

Dieses Phänomen beschränkt sich keineswegs allein auf die klinische Medizin, selbst in der exaktesten aller Naturwissenschaften, der Physik, können ähnliche Tendenzen nachgewiesen werden, wie KENNETH L. CANEVA 1978 gezeigt hat. Die Generation der um 1770 geborenen Physiker wie Paul ERMAN (1764-1851), Georg Gottlieb Schmidt (1768-1837), Thomas Johann Seebeck (17701831) oder GEORG WiLhELM MUNCKE (1772-1847) - die alle wesentliche Beiträge auf dem Gebiet der Elektrizitätslehre und des Magnetismus leisteten - betrachtete das Experiment eher unter qualitativen denn unter quantitativen Gesichtspunkten. Teilweise reflektierte ihre Grundeinstellung Newtons Ideal des „experimentum crucis“, indem der Forscher nach einem einfachen Experiment suchte, dessen Ergebnisse direkt und endgültig entweder für oder gegen eine bestimmte Behauptung sprechen sollten, ohne nach quantitativen Daten zu verlangen. Solche Experimente erbrachten typischerweise entweder gar keinen Effekt, oder sie bewiesen lediglich das Vorhandensein bzw. Nichtvorhandensein eines bestimmten Stoffes oder Phänomens.

Der wichtigste allgemeine Aspekt der Anschauungen der Physiker dieser Generation über das Verhältnis zwischen Erkenntnis und Experiment bestand in dem Glauben, das Experiment rangiere vor jeglicher Konzeptualisierung, was bedeutete, daß man das Experiment eher als eine Quelle denn als eine Kontrolle der Theorie auffaßte. Oft schien das Experiment sogar direkter Ausdruck eines grundlegenden Phänomens zu sein, was in Termini wie "Zentralphänomen“, „Hauptphänomen“ oder „Fundamentalversuch" gekleidet wurde. Eine Theorie mußte demgemäß vor allem "Anschaulichkeit" besitzen, „Intuition“ in der doppelten Wortbedeutung. Die andere Seite dieses Empirismus bestand in einer ausdrücklichen Ablehnung des Gebrauchs von Hypothesen in der Wissenschaft. Insbesondere empfanden es viele Physiker der älteren Generation als unstatthaft, Hypothesen vor dem Experiment zu bilden. Die Forderung, daß physikalisches Wissen anschaulich zu sein habe, wurde von einer Aversion gegen die Mathematik begleitet; numerische Daten wären für eine nicht-quantitative Theorie ja auch irrelevant gewesen, und es herrschte weitgehend Übereinstimmung darin, daß eine Theorie nicht anschaulich sein könne, wenn sie in der Sprache der Mathematik formuliert wurde. CANEVA nennt die Physik dieser Generation daher auch "science of the concrete“ oder „concretizing science“. Dies wäre sozusagen die deutsche Variante der empiristischen Tradition, die ihr Pendant in der englischen Naturphilosophie oder der französischen "physique expérimentale“ besal ${ }^{8}$. 


\section{Medizin im Umbruch}

Weit mehr noch als die Physik (und mit einer zeitlichen Verzögerung von etwa 20 Jahren) bleibt die Medizin unter den naturhistorisch orientieren Ärzten der Empirie und Beobachtung verhaftet. Man gewinnt den Eindruck, als sammelten die Wissenschaftler während der dreißiger und frühen vierziger Jahre akribisch Detail um Detail, ohne die Menge der Fakten jedoch - um es modern auszudrücken - „operationalisieren“ zu können. Gerade diese positivistische Suche nach bloßen "Thatsachen" stößt bei den Angehörigen der alten, noch von der Naturphilosophie geprägten Generation auf Unverständnis und Widerstand; viele von ihnen halten derartige Forschung schlicht für oberflächlich und seicht. Ein Beispiel für diese Einstellung bietet der Bonner Mediziner CHRISTIAN FRIEDRICH HARLESS (1773-1853), der als Epigone einer theoretisierenden, nicht-klinischen, spekulativ-naturphilosophischen Heilkunde gelten kann.

Auf der 20. Naturforscherversammlung 1842 in Mainz bringt Harless seine Abneigung gegen das Eindringen naturwissenschaftlicher Disziplinen und Arbeitsweisen in die Medizin deutlich zum Ausdruck; insbesondere Physik und Chemie dienen ihm dabei als Zielscheiben der Kritik. Im selben Jahr hat der um drei Jahrzehnte jüngere Chemiker Justus vON LIEBIG (1803-1873) sein Werk „Die organische Chemie in ihrer Anwendung auf Physiologie und Pathologie“ veröffentlicht und damit den Anspruch der exakten Naturwissenschaften auf Mitwirkung in der Medizin angemeldet. Der 69jährige HARLESS hingegen gebraucht in seinem Mainzer Vortrag harte Worte gegen die Überbewertung von Physik und Chemie in der Heilkunde: Sektiererei und Modesucht, Ballast und Flittergold sind die Vokabeln, mit denen er die neuesten Errungenschaften der Medizin belegt. Unwillkürlich erinnere ihn das gegenwärtige Treiben an die Iatrochemie des 17. Jahrhunderts, wenn auch in sublimerer Gestalt. Die Chemie überschätze ihre Macht in ungebührlicher Weise, wenn sie sich anmaße, die Erscheinungen des organischen Lebens bis hin zu den eigentümlichsten Äußerungen der höheren Animalität erklären zu wollen. Erregt fährt der Redner - mit deutlicher Wendung gegen LIEBIG - fort: „... wenn ... die Chemie mit solchen Uebergriffen sich der gesammten ... Lebenslehre bemächtigen will, und nur noch etwa sucht, wie sie auch den Geist und das Gemüth aus ihrer Offizin hervorgehen lasse, ... dann ist es ... an der Zeit, ... sich mit ... Entschiedenheit ... dagegen zu erklären ... damit die Physiologie und auch die Pathologie nicht endlich in der Chemie untergehen, wenn zumal die Autorität eines an Kenntnis und Scharfsinn ausgezeichneten Meisters in der Scheidekunst imponierend gegenüber steht ..."

Nicht sehr viel gnädiger kritisiert der Geheimrat die Anleihen der Medizin aus Physik, Mechanik und Optik; auch die ,jetzt so modern gewordene Microscopie" bedürfe einer reiflichen Prüfung; bei allem „Grossen und Wunderbaren, was sie uns schauen lässt, und was die Physiologie und Pathologie ... mit größter Beflissenheit aufnimmt, dürfte sie doch von Täuschung nicht frei sein und zu manchem Fehlschluss führen"10.

Offensichtlich befindet sich die Medizin in Deutschland zu Beginn der vierziger Jahre des 19. Jahrhunderts in einer methodologischen Krise, die nicht nur die Universitäten, sondern die gesamte Ärzteschaft erfaßt hat. So melden sich 
gerade jetzt die Praktiker mit methodischen Vorschlägen zu Wort. Auf der eben angeführten Mainzer Naturforscherversammlung von 1842 etwa wird der praktische Arzt LEONHARI) VON GIRGENSOHN (1784-1851) mit seiner „Methode, die praktische Medizin zu befördern" zitiert, die im Amtlichen Bericht in voller Länge abgedruckt ist. Der 58 Jahre alte GIRGENSOHN empfiehlt darin eine pathobiographische Nosologie, bei der man „an einem und demselben Subjecte die verschiedenartigsten Krankheiten" studieren müsse, die es im Laufe seines Lebens durchmache. Der Autor glaubt, daß weniger die Beschreibung ontologischer Krankheitseinheiten als vielmehr die individuelle Ausprägung eines Leidens beim einzelnen Patienten zum Haupthema einer künftigen Heilkunde werden sollte. GiRGENSOHN übt damit heftige Kritik an den Anhängern der Naturhistorischen Schule, die ihr Augenmerk stets auf die Klassifizierung des abstrakten Krankheitsorganismus richteten, während sie das individuelle Reaktionsmuster des Kranken als Störpotential aus ihren Betrachtungen zu eliminieren suchten. Andererseits hält er aber auch die Meinung der jüngeren Schule der „Physiologischen Heilkunde" um RoSER und WUNDERLICH für zu einseitig, nach deren Doktrin jede Krankheit nichts anderes als eine Funktionsstörung darstelle. Als Forschungsmethode empfiehlt der Praktiker GIRGENSOHN großangelegte Feldstudien in folgender Weise: „(Es) sollten Aerzte, welche schon längere Zeit in ihrem Wohnorte gewirkt haben, sich zu einem gemeinsamen Unternehmen verbinden und aus deren Praxis die lehrreichen Fälle zu pathologischen Lebensläufen auswählen, um sie in ein Archiv niederzulegen. Sind in diesem eine hinreichende Anzahl Beobachtungen gesammelt, so müsste eine Revision und Vergleichung derselben vorgenommen werden ... Alle Aufsätze würden an einen dazu zu erwählenden Sammler adressiert und endlich einem Commité übergeben werden, welches die gesammelten Erfahrungen ordnet, zweckmäßig umgestaltet und die für die Wissenschaft dienlichen Folgerungen daraus entnimmt"1!.

Die Hoffnung, durch bloße Zusammenstellung und Vergleichung eines angespeicherten Tatsachenmaterials zu fruchtbaren Theorien gelangen zu können, kennzeichnet den Weg der sogenannten "Analytischen Richtung" in der Medizin, die auf den Philosophen Etienne Bonnot DE Condillac (1715-1780) zurückgeführt werden kann. Condillac hatte in seinem 1780 erschienenen Werk "Logique, ou les premiers développements de l'art de penser" der Analyse eine grundlegende Rolle für alle Wissenschaften zugesprochen. Seine Ideen wurden in Frankreich von namhaften Ärzten aufgegriffen, die in Fortführung der analytischen Methode den Ehrgeiz hatten, die Medizin zu reformieren. Am programmatischsten trat GFORGES CABANIS (1757-1808) für sie ein, die konsequenteste Anwendung fand die Methode bei Philippe Pinel (1755-1826). Schon 1839 jedoch weist der 22jährige Mediziner und Philosoph HERMANN LotzE (18171881) in einer Rezension der "Allgemeinen Pathologie“ von KARL WiLHeLM STARK (1787-1845) auf den illusionären Ansatz der Analytischen Methode hin: Während für die Analytiker die Beobachtung Ausgangsbasis jeglicher Erkenntnis ist, hält LOTZE jene nicht für das primäre und wichtigste wissenschaftliche Instrument. Der theoretische Teil einer Wissenschaft, so meinte noch CABANIS, dürfe lediglich die Verkettung und Klassifikation sämtlicher Fakten eines Gebietes sein. Für LOTZE ist diese Art Abstraktion nur ein fragwürdiger Schritt aus der vorwissenschaftlichen Phase. Die Analytiker erkennen zwar den Kausalzusam- 
menhang an, glauben jedoch, daß dieser durch die bloße Beobachtung zustande kommt. Die Ursächlichkeit ist nach CABANIS nichts anderes als die sukzessive Ordnung der Ereignisse, die man durch Beobachtung feststellen kann. Damit wäre die Ätiologie der Krankheiten die Verallgemeinerung aus vergleichenden Krankengeschichten. Für LOTZE ist hingegen eine wissenschaftliche Medizin ohne Ätiologie im Sinne der Erklärung der Krankheit nicht möglich, sie bedarf der Gesetze und Theorien. Die Analytiker konnten diese Form der kausalen Erklärung nicht akzeptieren, da sie Gesetze mit Fakten gleichsetzten und jegliche Hypothesenbildung ablehnten, wie es schon CONDILLAC in seiner "Logik“ getan hatte ${ }^{12}$.

\section{Von Henle zu Virchow}

Als JAKOB HENLE (1809-1885) im Jahre 1844, damals noch Ordinarius für Anatomie und Physiologie in Zürich, den ersten Band seiner „Zeitschrift für Rationelle Medicin" herausgibt, versucht er in einem programmatischen Aufsatz mit dem Titel „Medizinische Wissenschaft und Empirie“ deren Grundzüge darzulegen. Für HENLE steht außer Zweifel, daß reine Empirie allein nicht ausreicht, um die Heilkunde in den Stand einer Wissenschaft zu erheben, jene erscheint ihm aber als Vorbedingung für exakte Forschung: „Immer werden die klinischen Beobachtungen oder an deren Stelle die Krankheitsbilder der speciellen Pathologie die feste Grundlage, gleichsam den Umriss bilden, dessen einzelne Theile die wandelbare Theorie ... weiter ausführt. Welche Symptome aus einem Complex zusammengehören, welche miteinander steigen und fallen, oder alterniren, wird immer zuerst empirisch auszumitteln sein, ehe eine Erklärung ihres innern Zusammenhanges versucht werden kann. Indem man aber darüber Hypothesen aufstellt und ihre Haltbarkeit im gegebenen Falle prüft, wird man nicht umhin können, die Erscheinungen selbst genauer in's Auge zu fassen; ausgerüstet mit Vorurtheilen, die uns nur nicht ans Herz gewachsen sein müssen, werden wir mehr und Manches richtiger sehen. Leider bestätigt sich nur zu oft der alte Spruch, dass dem, der durch das gefärbte Glas einer Theorie schaut, die Gegenstände farbig erscheinen, aber es ist eben so gewöhnlich, dass sie dem unbewaffneten Auge des sogenannten nüchternen Beobachters ganz entgehen. Jenes ist doch der Anfang einer Erkenntniss"13.

Mit diesen Worten rehabilitiert HENLE nicht nur den Wert der Theorie für den Erkenntnisprozeß, sondern er gibt auch dem Medium der Hypothese eine neue Funktion: Sie soll als bewußtes Vorurteil dienen, das jedoch dem Forscher „nicht ans Herz gewachsen" sein darf, sondern von ihm sehr nüchtern als etwas Vorläufiges, als Mittel zum Zweck betrachtet wird. Damit ist eine Neubewertung der Hypothese eingeleitet, die noch von den Vertretern der vorhin erwähnten älteren Physikergeneration weitgehend geringgeschätzt worden war, nämlich als schlechter Ersatz für fehlendes Wissen. Wenn Physiker wie etwa MUNCKE sie benutzt hatten, dann eher als nachträglichen Erklärungsversuch für ein Phänomen denn als präzise formulierten Satz, der durch ein erst folgendes Experiment zu überprüfen wäre. Auf diese Weise lassen sich Aufsatztitel verstehen wie jener, den MunCke 1819 im „Journal für Chemie und Physik“ (Band 25, S. 17-28) 
nannte: „Hypothesen zur Erklärung einiger räthselhafter Naturphänomene“. Jetzt, ein Vierteljahrhundert später, steht die Hypothese nicht mehr am Ende, sondern am Anfang des naturwissenschaftlichen Forschungsprozesses. Die Haltbarkeit der Hypothese müsse im gegebenen Falle geprüft werden, so sagt der Anatom HENLE, ohne jedoch über die Art und die näheren Bedingungen jener Prüfung genauere Auskunft zu geben. Seine Formulierung enthält in diesem Punkt noch eine spürbare Unschärfe, die der Konkretisierung bedarf.

Drei Jahre nach HENLE gründet 1847 der erst 26jährige Prosektor der Berliner Charité, Dr. RuDOLF VIRCHOw (1821-1902), ebenfalls eine neue medizinische Zeitschrift, das „Archiv für pathologische Anatomie und Physiologie und für klinische Medicin“. Der Pathologe VIRCHOw, zwölf Jahre jünger als der Anatom HENLE, hält bereits auf der Jahressitzung der Gesellschaft für wissenschaftliche Medicin zu Berlin am 20. Dezember 1847 einen Vortrag, den er unter dem Titel „Die naturwissenschaftliche Methode und die Standpunkte in der Therapie" im zweiten Band seiner Archivs (1849) publiziert. Zu diesem Zeitpunkt hat er gerade einen Ruf auf den Lehrstuhl für Pathologische Anatomie an der Universität Würzburg, den ersten seiner Art in Deutschland, angenommen.

VIRCHOW glaubt einleitend feststellen zu können, „daß während der letzten drei Decennien der denkende Theil der deutschen Aerzte die alte Brücke zwischen der Medicin und den übrigen Naturwissenschaften wieder aufzubauen bestrebt gewesen ist, und daß alle tonangebenden Schulen in Deutschland darin ... übereinstimmen, daß die Medicin im Range einer Naturwissenschaft, als Wissenschaft vom Menschen, als Anthropologie im weitesten Sinne, also ideal (prophetisch) als höchste Naturwissenschaft gefaßt werden müsse ... Suchen wir daher vorweg festzustellen, wie überhaupt die Naturwissenschaft gemacht wird, um daran die Möglichkeit der Construction der medicinischen Naturwissenschaft ... zu erkennen" "14. Den Geist der Naturwissenschaften sieht VIRCHOw in der naturwissenschaftlichen Methode begründet; er fühlt sich dabei in der Tradition von Francis Bacon (156I-1626), der ,zuerst mit Bewußtsein, nach einer langen Zeit des Träumens, die naturwissenschaftliche Methode gelehrt" habe ${ }^{15}$. Auf dieser Methode beruhen für ihn die Erfolge der großen Mediziner wie HaRvey, Haller, Bell, Magendie oder Johannes Müller. Sie sei „der Geist der Naturwissenschaften "16.

ViRCHOW beschreibt nun die naturwissenschaftliche Methode in einer klassischen Formulierung, die ich zunächst im ganzen zitieren möchte; sie lautet: „Die naturwissenschaftliche Methode, welche übrigens die einzige Methode ist, die überhaupt existiert, ... befähigt uns zunächst zur naturwissenschaftlichen Fragestellung ... Die naturwissenschaftliche Frage ist die logische Hypothese, welche von einem bekannten Gesetz durch Analogie und Induction weiterschreitet; die Antwort darauf giebt das Experiment, welches in der Frage selbst vorgeschrieben liegt. Jene Hypothese ist also das Facit einer Rechnung mit Thatsachen, und sie setzt daher eine umfassende Kenntniß der Thatsachen voraus; das Experiment ist das logisch nothwendige und vollkommen bewußte Handeln zu einem bestimmten Zweck. Jeder Mensch, der die Thatsachen kennt und richtig zu denken vermag, ist befähigt, die Natur durch das Experiment zur Beantwortung einer Frage zu zwingen, vorausgesetzt, daß er das Material besitzt, das Experiment einrichten zu können. Die Naturforschung setzt also Kenntniß der Thatsachen, 
logisches Denken und Material voraus; diese drei, in methodischer Verknüpfung, erzeugen die Naturwissenschaft. Alle Kenntniß der Thatsachen, ist eine historische, ... insofern man nur das genau weiß, was man historisch weiß. Die nackten Thatsachen sind zweifelhafte Waffen; es ist nothwendig, daß man weiß, wie sie erhärtet sind, um ihre Stärke zu kennen. Die Medicin aber bedarf einer historischen Kenntniß mehr, als jede andere Wissenschaft ..."

Soweit ViRCHOWs Erläuterung der naturwissenschaftlichen Methode aus dem Jahre 1849. Hatte er schon die Medizin als die höchste Naturwissenschaft, als „Anthropologie im weitesten Sinne“ beschrieben, so beansprucht er auch für ihre Methode universalen Charakter. Sie ist die einzige Methode überhaupt! Mit ihrer Hilfe gelingt es, von einem Naturgesetz zum nächsten voranzuschreiten und so den Bestand gesicherten Wissens kontinuierlich zu vermehren. Der Weg dorthin führt über die logisch entwickelte Hypothese, die man durch Analogie und Induktion gewinnt. Nur scheinbar ist mit dem Analogieschluß die romantische Naturforschung rehabilitiert, denn jener liefert für den Naturwissenschaftler nicht mehr die Lösung eines Problems, sondern nur noch die Fragestellung. Die Antwort kann erst das Experiment geben, das VIRCHOw als ein Iogisch notwendiges und rational durchkalkuliertes Handeln zu einem bestimmten Zweck definiert. Voraussetzung für das Experiment ist aber dreierlei: eine umfassende Kenntnis der Tatsachen, logisches Denkvermögen und geeignetes Material für die Durchführung des Versuchs. Sofern man diese Prämissen erfüllt, kann man die Natur zur Beantwortung jeder beliebigen Frage zwingen.

Die umfassende Kenntnis der Tatsachen muß auf historisch gesichertem Wissen beruhen; nur was man historisch weiß, weiß man genau! Alle Kenntnis stamme aus sinnlicher Beobachtung und müsse sich ständig an dieser bewähren. In diesem Punkt bekennt sich VirCHOW zum Sensualismus JOHN LOCKES (1632-1704); denn der Naturwissenschaftler „kennt ... nur das, was der naturwissenschaftlichen (sinnlichen) Forschung zugänglich ist ${ }^{\text {"18 }}$.

Mit diesen Leitlinien ist das Programm der naturwissenschaftlichen Medizin umrissen, das in den folgenden Jahrzehnten Zug um Zug realisiert wird. Vor allem die theoretischen Grundlagenfächer wie Anatomie, Physiologie und Pathologie begründen die internationale Führungsrolle der deutschen Medizin in der zweiten Hälfte des 19. Jahrhunderts. Diese Entwicklung dokumentiert sich auch im institutionellen Rahmen: Zwischen 1849 und 1876 etabliert sich die Pathologische Anatomie mit Lehrstühlen an allen deutschen Universitäten, während sich im gleichen Zeitraum fast überall die Physiologie als autonomes Fach von der Anatomie abtrennt; als letzte Hochschule erhält 1891 die Universität Gießen ein eigenes Ordinariat für Physiologie.

\section{Die Grenzen der naturwissenschaftlichen Methode}

Die Ziele, die Rudolf VIRCHow mit der naturwissenschaftlichen Methode zu erreichen hofft, erschöpfen sich allerdings nicht in der Erweiterung des biologisch-medizinischen Wissens. Als eine soziale, eine umfassend anthropologische Wissenschaft soll die Heilkunde in allen Dimensionen auch des gesellschaftlichen und politischen Lebens wirksam werden. Dieser utopische Entwurf kulmi- 
niert in VIRCHOws Rede auf der Rostocker Naturforscherversammlung im September 1871, wenige Monate nach der Reichsgründung BISMARCKs, zum Thema „Aufgaben der Naturwissenschaften in dem neuen nationalen Leben Deutschlands“. Der heraufziehende Kulturkampf kündigt sich an, als der inzwischen weltberühmte Berliner Pathologe die katholische Kirche heftig attackiert: „Jeder Fortschritt, den eine Kirche in dem Aufbau ihrer Dogmen macht, führt zu einer ... Bändigung des freien Geistes; jedes neue Dogma ... verengt den Kreis des freien Denkens ... Die Naturwissenschaft umgekehrt befreit mit jedem Schritte ihrer Entwicklung, sie eröffnet dem Gedanken neue Bahnen ... und man kann wohl hoffen, dass es gelingen werde, in dem Fortschreiten des Wissens auch zugleich ein Motiv höheren sittlichen Eifers, eine Quelle immer grösseren Strebens nach Wahrheit, Ehrlichkeit und Treue im Handeln zu finden ... Wern es gelingt, unsere Methode zu der Methode der ganzen Nation zu machen, sie nicht blos in immer grösserer Ausdehnung den materiellen Arbeitsleistungen zu Grunde zu legen, sondern sie ... zu erheben zu der eigentlichen Maxime des Denkens, des sittlichen Handelns, so wird die wahre Einheit der Nation gewonnen sein"19. Zwei Jahre später, auf der Wiesbadener Versammlung von 1873 , setzt ViRCHOW diese Betrachtungen unter dem Titel „Die Naturwissenschaften in ihrer Bedeutung für die sittliche Erziehung der Menschheit" fort. In dieser Rede fordert er, daß ,Jeder von seinem Standpunkt ... helfe, die Moral als eine empirische Wissenschaft nach den Regeln zu entwickeln, welche die allgemeine Naturwissenschaft constituirt hat ${ }^{20}$.

Am gleichen Ort, nämlich ebenfalls in Wiesbaden, jedoch 85 Jahre später, auf der 100. Naturforscherversammlung 1958, hat KARL JASPERS (1883-1969) zum Verhältnis von Wissenschaft und Philosophie Stellung genommen. Mit Blick auf die Entwicklung im 19. und 20. Jahrhundert meinte JASPERS, die Philosophie habe, ihrer selbst nicht mehr gewiß, versucht, sich als exakte Wissenschaft zu konstituieren. „Dabei ging ... sie sich selbst verloren in der Fiktion einer, wissenschaftlichen Philosophie', die bis heute fortdauert. Von der anderen Seite ließen viele Träger wissenschaftlicher Forschung - unwissenschaftlich = ihre Erkenntnis zum Weltbild, das Wissen von ihrer Methode zur Erkenntnistheorie überhaupt, ihre Gesamtanschauung zum Wechselbalg der sogenannten wissenschaftlichen Weltanschauung werden“"2!.

Diese Kritik des Mediziners und Philosophen Jaspers verdeutlicht die Grenzen der naturwissenchaftlichen Methode, die viele Forscher im späten 19. Jahrhundert glaubten überwinden zu können oder die sie einfach nicht erkannten. Zwar sah schon RuDOLF VIRCHOW 1871, daß die deutschen Hochschulen und Universitäten einen Einfluß auf den Gang des Deutsch-Französischen Krieges gehabt hätten und daß die deutsche Wissenschaft, indem sie eine so große Zahl von Ingenieuren, Fabrikanten und Produzenten heranbildete, eine entscheidende Einwirkung auf die Kriegsführung ausgeübt habe ${ }^{22}$, doch diesen Zusammenhang vermerkte der große Gelehrte noch voller Stolz auf die soeben erlangte deutsche Einheit. Und der Physiker QuinckE schloß seine Eröffnungsansprache der Naturforscherversammlung von 1889 mit den Worten: „Die jetzt glücklich errungene Einheit der Nation erscheint uns um so wertvoller, je grössere Schwierigkeiten zu überwinden waren, ehe der Bau vollendet dastand, von den lebendigen Mauern der allgemeinen Wehrpflicht geschützt. Gemeinsam haben 
Praxis und Wissenschaft, das deutsche Volk und seine Fürsten daran gearbeitet gemeinsam werden sie weiter für seine Erhaltung sorgen ${ }^{23}$.

Nur 25 Jahre danach, am I. August 1914, wurde offenkundig, daß die Gelehrten des 19. Jahrhunderts die Kraft der naturwissenschaftlichen Methode überschätzt hatten. Sie, die sich als nützliches Hilfsmittel für den wissenschaftlichen Fortschritt bewährte und bis heute bewährt, mußte an der Aufgabe, ein reformatorisches Medium auch für Politik und Gesellschaft zu werden, scheitern. Dieses Versagen war nicht die Schuld der Methode, es erweist sich vielmehr als eine Folge inadäquater Anwendung auf Gebieten, die sich der experimentellen Nachprüfung prinzipiell entziehen. RUDOLF ViRCHOWs Traum von der Konstituierung der Gesellschaft auf physiologischer Grundlage blieb - so mitreißend er auch konzipiert war - eine Utopie des 19. Jahrhunderts. Geblieben ist uns - am Ende des 20. Jahrhunderts - die naturwissenschaftliche Methode und ihr erfolgreicher Einsatz in der Medizin, der um so klarer hervortreten kann, je mehr wir uns auch die Grenzen dieses Verfahrens vergegenwärtigen.

\section{Anmerkungen}

1 Amtlicher Bericht 1829, S. 15

2 ibid., S. 16

3 ibid., S. 17

4 ibid., S. 31

5 Tageblatt 1889, S. 42

'ibid., S. 43

7 Rothschuh 1968, S. 186 f.

s Caneva 1978, S. 64-71

2Amtlicher Bericht 1842, S. 88

10 ibid., S. 289

11 ibid., S. 297-298

12 Vgl. Tsouyopoulos/Bleker 1976

${ }_{13}$ Henle 1844, S. 34-35

${ }_{14}$ Virchow 1849, S. 6

15 ibid., S. 7

${ }^{16}$ loc.cit.

17 ibid., S. 7-8

18 ibid., S. 9

19 Tageblatt 1871, S. 81

20) Tageblatt 1873, S. 207

21 Jaspers 1986, S. 55

2. Tageblatt 1871, S. 76. Vgl. auch Bauer 1985, S. 77

23 Tageblatt 1889, S. 45

\section{Literatur}

Amtlicher Bericht über die Versammlung deutscher Naturforscher und Ärzte in Heidelberg im September 1829 (1829) erstattet von den damaligen Geschäftsführern F. Tiedemann und L. Gmelin. Heidelberg

Amtlicher Bericht über die 20. Versammlung der Gesellschaft deutscher Naturforscher und Aerzte zu Mainz im September 1842 (1843) Hrsg. von den Geschältsführern derselben, Gröser u. Bruch. Mainz 
Bauer A (1985) Pathologie auf den Versammlungen Deutscher Naturforscher und Ärzte von 1822 bis 1872. Die Krankheitslehre auf dem Weg zur naturwissenschaftlichen Morphologie. Med. Habilitationsschrift, Heidelberg

Bleker J (1981) Die naturhistorische Schule 1825-1845. Ein Beitrag zur Geschichte der klinischen Medizin in Deutschland. Stuttgart

Caneva KL (1978) From galvanism to electrodynamics: The transformation of German physics and its social context. In: McCormmach R, Pyenson L (Eds) Historical studies in the Physical Sciences 9:63-159, Baltimore/London

Henle J (1844) Medizinische Wissenschaft und Empirie. Z Rationelle Med 1:1-35

Jaspers K (1986) Der Arzt im technischen Zeitalter. Vortrag, gehalten auf der 100. Tagung der Gesellschaft Deutscher Naturforscher und Ärzte 1958 in Wiesbaden. Wiederabgedr. in: Jaspers K, Der Arzt im technischen Zeitalter. München Zürich, S 39-58

Rothschuh KE (1968) Deutsche Biedermeiermedizin, Epoche zwischen Romantik und Naturalismus (1830-1850). Gesnerus 25:167-187

Rothschuh KE (1978) Konzepte der Medizin in Vergangenheit und Gegenwart. Stuttgart

Tageblatt der 44. Versammlung Deutscher Naturforscher und Ärzte in Rostock vom 18. bis 24. Sept. 187I (1871). Unter Verantwortung des Redactions-Comités. Rostock.

Tageblatt der 46. Versammlung Deutscher Naturforscher und Aerzte in Wiesbaden vom 18. bis 24. Sept. 1873 (1873). Wiesbaden.

Tageblatt der 62. Versammlung Deutscher Naturforscher und Ärzte in Heidelberg vom 18. bis 23. Sept. 1889 (1890). Heidelberg.

Tsouyopoulos N, Bleker J (1976) Über analytische und synthetische Methode in der Medizin des 19. Jahrhunderts. In: Acta Congressus Internationalis XXIV Historiae Artis Medicinae, 25-31 Augusti 1974 Budapestini, tom. I. Budapest, S 49-55

Virchow R (1849) Die naturwissenschaftliche Methode und die Standpunkte in der Therapie. Arch Pathol Anat Physiol Klin Med 2:3-37 